I 78 and 180 with some 182 , and has no odd isotopes. Tantalum is 181 and $I 83$, tungsten 184 only, and element 75 would have 185 or 187 if they existed. Iridium appears to have I9I as well as I93; platinum has I94 and I96, possibly I92, but 198 is unlikely. Mercury is 198, I99, 200, 202, and 204 and not 201 as Aston finds, but I cannot add I97 as he thinks possible.

Gold is 197 and simple if Aston is right about mercury; otherwise it should have I99 also. Thallium is 203 and 205 , lead principally 208 and 206, the former in excess. Bismuth is simple and 209. Polonium is of course $2 \mathrm{IO}$, and the only member of element 84 with a chance (and that a very remote one) of being isolated. Thorium is simple and 232. One isotope of element 89 is too unstable ever to be isolated. Element $9 \mathrm{I}$ has $23 \mathrm{I}$ and 233, the former being probably protoactinium. Uranium, which is complex, has been discussed in my paper in the Philosophical Magazine.

The order of intensity of the isotopes cannot be given accurately from these considerations, but a rough sorting into major and minor isotopes is not difficult to make. Mass-numbers which belong to no atomic number are difficult to estimate. At present I feel sure of ten even ones and thirty-one odd below polonium, most of which Aston has found. All but one of the former are of the form $4 n+2$, and more than three-fifths of the latter of the form $4 n+\mathbf{r}$. There appear to be at least thirty simple elements if my predictions be added to Aston's certainties. Fourteen of these have accepted atomic weights within 0.05 of a unit, and as many fall short of a unit by this amount as exceed it. I have assumed Aston's whole-number rule in all the numbers given in this letter.

After these mass-numbers had been deduced I found that the complexity of an element was apparently a simple function of the atomic number I6n. Thus there is a probability that elements of atomic numbers $\mathrm{I} 6 n+7, \mathrm{I} 6 n+10$, and $\mathrm{I} 6 n+\mathrm{I}$ I are simple; that $\mathrm{x} 6 n+3$, $16 n+5,16 n+13$, and $16 n+I_{5}$ have two isotopes; that $I 6 n+8$ and $I 6 n+I_{4}$ have no odd isotopes; and that $16 n, 16 n+2$, and $16 n+12$ have odd isotopes. If this deduction be substantiated by experimental work it should throw light on the constitution and stability of the nucleus.

Dr. Lee's Laboratory, Christ Church, Oxford, October 3, I923.

\section{The Measurement of Very High Temperature.}

IN I9I4 Lummer ${ }^{1}$ described some experiments on an arc burning in a gas at high pressure. His method of determining the temperature based on the increase of surface brightness of the positive crater is extremely unsatisfactory, and his figures, using his own values of surface brightness, appear to be nearly three thousand degrees too low. It seemed desirable, therefore, to repeat and extend the experiments and determine the temperature more precisely. A very accurate way of doing this would be to determine the ratio of the intensity of the light at two wave-lengths as far as possible apart, which would define the temperature if the positive crater were a complete radiator. This assumption need not be made if ratios of the intensities are determined at two different temperatures, one of which is known. Thus, for example, in the region in which Wien's law holds, if $a_{\lambda}$ is a constant proportional to the emissivity, the intensity is given by

${ }^{1}$ Lummer, "Verflüssigung der Kohle und Herstellung der Sonnentemperatur." (Sammlung Vieweg.)

NO. 28 I 6 , VOL. I I 2$]$

$$
\mathrm{E}_{\lambda \mathrm{T}}=\frac{\alpha_{\lambda} \cdot e^{-c / \lambda \mathrm{T}}}{\lambda^{5}}
$$

and

$$
\log \frac{E_{\lambda_{1} T_{2}} \cdot E_{\lambda_{2} T_{2}}}{E_{\lambda_{1} T_{2}} \cdot E_{\lambda_{2} T_{1}}}=c\left(\frac{I}{\lambda_{1}}-\frac{I}{\lambda_{2}}\right)\left(\frac{I}{T_{1}}-\frac{I}{T_{2}}\right),
$$

which determines $T_{2}$ in terms of $T_{1}$. Since $a_{\lambda}$, which may also contain the sensitivity of the measuring instrument, disappears from the final equation, this method is very convenient and may be made very accurate.

Two methods were used for determining the intensity, one by the use of a wedge as suggested by Prof. Merton, ${ }^{2}$ the other making use of the photoelectric effect. The first method is more convenient in many cases ; the second is probably more accurate.

The main difficulty is to make sure that one is really observing the hottest part of the crater. It is very difficult to keep the arc constant at high pressures, and obviously too low a temperature will be found if the arc shifts during the exposure so that part of the measurement is carried out on the colder parts surrounding the crater. If this has been avoided, comparison of the intensity at any two wave-lengths at atmospheric pressure and at high pressure enables the temperature at the high pressure to be calculated in terms of the known temperature of the normal arc. A check in the method is given by the constancy of the temperature found using various wave-lengths. The divergence from the mean is within the limits of experimental error.

Owing to the difficulty outined above, observations at the same pressure do not repeat very accurately, though the highest values are fairly consistent. The following table summarises the provisional results for an arc in nitrogen:

\begin{tabular}{|c|c|}
\hline $\begin{array}{c}\text { Pressure in } \\
\text { Atmospheres. }\end{array}$ & Temperature. \\
\hline & \\
I & $(4 \mathrm{r} 9 \mathrm{O}) \mathrm{A}$ \\
6 & 4680 \\
I8 & $6 \mathrm{r} 80$ \\
33 & 6520 \\
80 & 8620 \\
\hline
\end{tabular}

As already stated, these are minimum temperatures; and indications on one plate $\left(10,000^{\circ}\right.$ at $5^{\circ}$ atmospheres) seem to justify the suspicion that they may be considerably underestimated.

Further experiments making use of a number of improved methods are now in progress, and it is hoped shortly in a fuller publication to give more accurate values for the temperature of the crater as a function of the pressure and nature of the gas. The fact seems certain, however, that one can by this means reach temperatures in the laboratory considerably higher than the temperature at the surface of the sun.

It may be interesting to note here the strong reversal of some of the cyanogen bands shown on the plates within certain limits of pressure and temperature. The phenomenon is most noticeable between 30 and 40 atmospheres, and it should be possible to locate these limits more definitely in the course of the experiments.

I have in conclusion to acknowledge a deep debt of gratitude to Prof. Lindemann for much helpful criticism and encouragement.

Clarendon Laboratory, Oxford, September 22.

2 Merton and Nicholson (Phil. Trans. Roy. Soc. A. 2I7). Prof. Merton kindly lent me the spectrometer and wedge which he used in his own investigations, and I take this opportunity of thanking him for the loan of the apparatus and for his assistance in initiatin me into the details of his the apparat
method. 